\title{
AN EXACT APPROACH FOR THE SINGLE MACHINE SCHEDULING PROBLEM WITH LINEAR EARLY AND QUADRATIC TARDY PENALTIES
}

\author{
JORGE M. S. VALENTE \\ LIAAD, Faculdade de Economia \\ Universidade do Porto, Portugal \\ jvalente@fep.up.pt
}

Received 29 December 2006

Accepted 11 March 2007

\begin{abstract}
In this paper, we consider the single machine scheduling problem with linear earliness and quadratic tardiness costs, and no machine idle time. We propose a lower bounding procedure based on the relaxation of the jobs' completion times. Optimal branch-andbound algorithms are then presented. These algorithms incorporate the proposed lower bound, as well as an insertion-based dominance test.

The branch-and-bound procedures are tested on a wide set of randomly generated problems. The computational results show that the branch-and-bound algorithms are capable of optimally solving, within reasonable computation times, instances with up to 20 jobs.
\end{abstract}

Keywords: Scheduling; single machine; linear earliness; quadratic tardiness; lower bound; branch-and-bound.

\section{Introduction}

In this paper, we consider a single machine scheduling problem with linear earliness and quadratic tardiness costs, and no machine idle time. Single machine scheduling environments actually occur in many practical operations (for a specific example in the chemical industry, see Wagner et al. (2002)). Also, the performance of production environments is frequently determined by a single bottleneck machine. Single processor models are then quite useful for scheduling such a machine. Moreover, results and insights obtained for single machine problems can often be applied to more complex scheduling environments, such as flow shops or job shops.

Scheduling models with earliness and tardiness penalties are compatible with a recent trend in industry, namely the adoption of supply chain management by many organizations. In this approach, customers and suppliers try to integrate the flow of materials, in order to improve the efficiency of the supply chain and provide a better service to the end user. The adoption of supply chain management has caused 
organizations to view both early and tardy deliveries as undesirable. Early/tardy scheduling models are also suited to the philosophy of just-in-time (JIT) production. In fact, the JIT production philosophy emphasizes producing goods only when they are needed. Therefore, in a JIT setting an ideal schedule is one in which all jobs are completed exactly on their due dates.

In this paper, we consider linear earliness and quadratic tardiness costs. On the one hand, early deliveries or early completions of jobs result in unnecessary inventory that ties up cash, as well as space and resources required to maintain and manage the inventory. These costs tend to be proportional to the quantity of inventory held, and therefore a linear penalty is used for early jobs.

Late deliveries, on the other hand, can result in lost sales and loss of customer goodwill, and may also cause disruptions in stages further down the production line or supply chain. We consider a quadratic tardiness penalty, instead of the more usual linear tardiness or maximum tardiness functions. As described in Sun et al. (1999), the quadratic penalty may be selected over these two other tardiness measures for the following reasons.

First, the maximum tardiness performance measure does not distinguish between schedules where tardiness occurs for all jobs, or only one, as long as the maximum tardiness is the same. Second, when the linear tardiness function is used, it is possible that a single or only a few jobs contribute the majority of the cost, without regard to how the overall tardiness is distributed. In fact, the linear tardiness criterion does not differentiate between sequences where all jobs are only a little tardy, or a single job is extremely late, providing the total cost is equal. The quadratic penalty overcomes these problems, and provides a more robust performance measure. Moreover, a quadratic tardiness penalty is also appropriate in practice. In fact, tardiness represents an important attribute of service quality. Also, as proposed in the loss function of Taguchi (1986), a customer's dissatisfaction tends to increase quadratically with the tardiness.

We assume that no machine idle time is allowed. This assumption is actually appropriate for many production settings. In fact, when the capacity of the machine is limited when compared with the demand, the machine must indeed be kept running in order to satisfy the customers' orders. Also, the assumption of no idle time is justified when the machines have high operating costs. Furthermore, idle time must also be avoided when starting a new production run involves high setup costs or times. Some specific examples of production settings where the no idle time assumption is appropriate, have been given by Korman (1994) and Landis (1993).

Formally, the problem we consider can be stated as follows. A set of $n$ independent jobs $\left\{J_{1}, J_{2}, \ldots, J_{n}\right\}$ has to be scheduled on a single machine that can handle at most one job at a time. The machine is assumed to be continuously available from time zero onwards, and preemptions are not allowed. Job $J_{j}, j=1,2, \ldots, n$, requires a processing time $p_{j}$ and should ideally be completed on its due date $d_{j}$. For a given schedule, the earliness and tardiness of $J_{j}$ are respectively defined as $E_{j}=\max \left\{0, d_{j}-C_{j}\right\}$ and $T_{j}=\max \left\{0, C_{j}-d_{j}\right\}$, where $C_{j}$ is the completion time of $J_{j}$. The objective is then to find a schedule that minimizes the sum of linear 
earliness and quadratic tardiness costs $\sum_{j=1}^{n}\left(E_{j}+T_{j}^{2}\right)$, subject to the constraint that no machine idle time is allowed.

This problem has been previously considered by Valente (2006). He presented several dispatching heuristics, and analyzed their performance on a wide range of instances. Schaller (2004) considered the corresponding problem with inserted idle time. He presented a timetabling procedure to optimally insert idle time in a given sequence, as well as a branch-and-bound procedure and simple and efficient heuristics.

The single machine early/tardy problem with linear earliness and tardiness costs $\sum_{j=1}^{n}\left(E_{j}+T_{j}\right)$ has also been previously considered by Garey et al. (1988) and Kim and Yano (1994). Garey et al. (1988) showed that the problem is NP-hard, and proposed a timetabling procedure. Kim and Yano (1994) presented some properties of optimal solutions, and developed both optimal and heuristic algorithms.

The minimization of the quadratic lateness $\sum_{j=1}^{n} L_{j}^{2}$, where the lateness of $J_{j}$ is defined as $L_{j}=C_{j}-d_{j}$, has also been previously considered. Gupta and Sen (1983) presented a branch-and-bound algorithm and a heuristic rule for the problem with no idle time. Su and Chang (1998) and Schaller (2002) considered the insertion of idle time, and proposed timetabling procedures and heuristic algorithms. Sen et al. (1995) presented a branch-and-bound algorithm for the weighted problem $\sum_{j=1}^{n} w_{j} L_{j}^{2}$ where idle time is allowed only prior to the start of the first job. Baker and Scudder (1990) provide an excellent survey of scheduling problems with earliness and tardiness penalties, while Kanet and Sridharan (2000) give a review of scheduling models with inserted idle time that complements our focus on a problem with no machine idle time.

In this paper, we first develop a lower bounding procedure. An optimal branchand-bound algorithm that incorporates this lower bound, as well as an insertionbased fathoming test, is then proposed. The branch-and-bound procedure is then tested on a wide set of randomly generated problems.

The remainder of this paper is organized as follows. The lower bounding procedure is described in Sec. 2. In Sec. 3, we discuss the implementation details of the branch-and-bound algorithm. The computational results are presented in Sec. 4 . Finally, some concluding remarks are given in Sec. 5.

\section{Lower Bounding}

In this section, we first propose a lower bounding procedure based on a relaxation of the completion times. Then, we present special cases in which the lower bounding procedure provides the optimum objective function value. Finally, we discuss the generalization and applicability of the lower bounding technique to other objective functions.

\subsection{The lower bounding procedure}

In this section, we propose a lower bound for the linear earliness/quadratic tardiness problem. For convenience, and without loss of generality, it will be assumed 
throughout this section that we wish to calculate a lower bound for a sequence that starts at time $t=0$. This makes the presentation of the lower bound easier and clearer, and the extension of the lower bound to partial sequences that start at any time $t>0$ is straightforward.

Let $Z$ denote the objective function of the considered problem, i.e. $Z=$ $\sum_{j=1}^{n}\left(E_{j}+T_{j}^{2}\right)=\sum_{j=1}^{n} \max \left(d_{j}-C_{j}, 0\right)+\sum_{j=1}^{n}\left[\max \left(C_{j}-d_{j}, 0\right)\right]^{2}$. Also, let $[j]$ denote the job in the $j$ th position in a sequence. In order to derive the lower bound, we consider a relaxation of the completion times $C_{j}$. Let $C_{l}^{\mathrm{LPT}}$ be the sum of the processing times of the first $l$ jobs, when the jobs are ordered in longest processing time (LPT) order (i.e. $p_{[j]} \geq p_{[k]}$ for $j<k$ ). Similarly, let $C_{l}^{\mathrm{SPT}}$ be the sum of the processing times of the first $l$ jobs, when the jobs are ordered in shortest processing time (SPT) order (i.e. $p_{[j]} \leq p_{[k]}$ for $j<k$ ).

We can now define a modified objective function $Z^{\prime}$ by replacing the completion times $C_{j}$ in $Z$ with $C_{l}^{\mathrm{LPT}}$ and $C_{l}^{\mathrm{SPT}}$. More precisely, the modified objective function $Z^{\prime}$ is defined as $Z^{\prime}=\sum_{j=1}^{n} \max \left(d_{[j]}-C_{j}^{\mathrm{LPT}}, 0\right)+\sum_{j=1}^{n}\left[\max \left(C_{j}^{\mathrm{SPT}}-d_{[j]}, 0\right)\right]^{2}$. For any given sequence, we have $Z^{\prime} \leq Z$, since $C_{j}^{\mathrm{LPT}} \geq C_{[j]}$ and $C_{j}^{\mathrm{SPT}} \leq C_{[j]}$. Let $d_{j}^{\mathrm{EDD}}$ denote the due date of the $j$ th job, when the jobs are ordered in earliest due date (EDD) order (i.e. $d_{[j]} \leq d_{[k]}$ for $\left.j<k\right)$. Finally, let $Z_{\mathrm{EDD}}^{\prime}=\sum_{j=1}^{n} \max \left(d_{j}^{\mathrm{EDD}}-\right.$ $\left.C_{j}^{\mathrm{LPT}}, 0\right)+\sum_{j=1}^{n}\left[\max \left(C_{j}^{\mathrm{SPT}}-d_{j}^{\mathrm{EDD}}, 0\right)\right]^{2}$.

Theorem 2.1. $Z_{\mathrm{EDD}}^{\prime} \leq \min Z$, i.e. $Z_{\mathrm{EDD}}^{\prime}$ is a lower bound for the optimal objective function value of the linear earliness/quadratic tardiness problem.

Proof. As previously mentioned, we have $Z^{\prime} \leq Z$ for any specific sequence. Therefore, it is sufficient to prove that $Z_{\mathrm{EDD}}^{\prime}=\min Z^{\prime}$, since we then have $Z_{\mathrm{EDD}}^{\prime} \leq Z$ for all possible sequences. This will be done by contradiction. Consider a schedule $S$ in which jobs $j$ and $i$ are scheduled in positions $l_{1}$ and $l_{2}$, respectively, with $l_{1}<l_{2}$ and $d_{j}>d_{i}$. Therefore, jobs $i$ and $j$ are not scheduled in EDD order. Also consider a schedule $S^{\prime}$ that is identical to $S$, except for the fact that the positions of jobs $i$ and $j$ have been interchanged. We must show that $Z^{\prime}\left(S^{\prime}\right) \leq Z^{\prime}(S)$.

Since only the positions of jobs $i$ and $j$ are different in schedules $S$ and $S^{\prime}$, all other jobs $k$, with $k \neq i, j$, occupy the same positions in both schedules. The contribution of those jobs $k$ to $Z^{\prime}$ is therefore identical in schedules $S$ and $S^{\prime}$. Hence, it suffices to compare the contributions of jobs $i$ and $j$ to $Z^{\prime}$.

We first consider the change that may occur in the first part of $Z^{\prime}$, i.e. the change in the term $\sum_{j=1}^{n} \max \left(d_{[j]}-C_{j}^{\mathrm{LPT}}, 0\right)$. Let $E(S)$ denote the sum of the contributions of jobs $i$ and $j$ to the term $\sum_{j=1}^{n} \max \left(d_{[j]}-C_{j}^{\mathrm{LPT}}, 0\right)$ in schedule $S$. Therefore, we have $E(S)=\max \left(d_{j}-C_{l_{1}}^{\mathrm{LPT}}, 0\right)+\max \left(d_{i}-C_{l_{2}}^{\mathrm{LPT}}, 0\right)$ and $E\left(S^{\prime}\right)=$ $\max \left(d_{i}-C_{l_{1}}^{\mathrm{LPT}}, 0\right)+\max \left(d_{j}-C_{l_{2}}^{\mathrm{LPT}}, 0\right)$. Also let $\Delta E=E\left(S^{\prime}\right)-E(S)$. The following three cases must then be considered.

Case 1. $C_{l_{2}}^{\mathrm{LPT}}<d_{i}<d_{j}$. We have $E(S)=d_{j}-C_{l_{1}}^{\mathrm{LPT}}+d_{i}-C_{l_{2}}^{\mathrm{LPT}}$ and $E\left(S^{\prime}\right)=$ $d_{i}-C_{l_{1}}^{\mathrm{LPT}}+d_{j}-C_{l_{2}}^{\mathrm{LPT}}$. Therefore, $\Delta E=0$. 
Case 2. $d_{i} \leq C_{l_{2}}^{\mathrm{LPT}} \leq d_{j}$. In this case, we have $E(S)=d_{j}-C_{l_{1}}^{\mathrm{LPT}}$ and $E\left(S^{\prime}\right)=$ $\max \left(d_{i}-C_{l_{1}}^{\mathrm{LPT}}, 0\right)+d_{j}-C_{l_{2}}^{\mathrm{LPT}}$. We then have $\Delta E=\max \left(d_{i}-C_{l_{1}}^{\mathrm{LPT}}, 0\right)+$ $C_{l_{1}}^{\mathrm{LPT}}-C_{l_{2}}^{\mathrm{LPT}}=\max \left(d_{i}, C_{l_{1}}^{\mathrm{LPT}}\right)-C_{l_{2}}^{\mathrm{LPT}}$. Therefore, $\Delta E \leq 0$, since $d_{i} \leq$ $C_{l_{2}}^{\mathrm{LPT}}$ and $C_{l_{1}}^{\mathrm{LPT}}<C_{l_{2}}^{\mathrm{LPT}}$.

Case 3. $d_{i}<d_{j}<C_{l_{2}}^{\mathrm{LPT}}$. We have $E(S)=\max \left(d_{j}-C_{l_{1}}^{\mathrm{LPT}}, 0\right)$ and $E\left(S^{\prime}\right)=\max \left(d_{i}-\right.$ $\left.C_{l_{1}}^{\mathrm{LPT}}, 0\right)$. Consequently, $\Delta E=\max \left(d_{i}-C_{l_{1}}^{\mathrm{LPT}}, 0\right)-\max \left(d_{j}-C_{l_{1}}^{\mathrm{LPT}}, 0\right)$. Therefore, we have $\Delta E \leq 0$, since $d_{i}<d_{j}$.

We now consider the change in the second part of $Z^{\prime}$, i.e. the change in the term $\sum_{j=1}^{n}\left[\max \left(C_{j}^{\mathrm{SPT}}-d_{[j]}, 0\right)\right]^{2}$. Let $T(S)$ denote the sum of the contributions of jobs $i$ and $j$ to the term $\sum_{j=1}^{n}\left[\max \left(C_{j}^{\mathrm{SPT}}-d_{[j]}, 0\right)\right]^{2}$ in schedule $S$. We then have $T(S)=\left[\max \left(C_{l_{1}}^{\mathrm{SPT}}-d_{j}, 0\right)\right]^{2}+\left[\max \left(C_{l_{2}}^{\mathrm{SPT}}-d_{i}, 0\right)\right]^{2}$ and $T\left(S^{\prime}\right)=\left[\max \left(C_{l_{1}}^{\mathrm{SPT}}-\right.\right.$ $\left.\left.d_{i}, 0\right)\right]^{2}+\left[\max \left(C_{l_{2}}^{\mathrm{SPT}}-d_{j}, 0\right)\right]^{2}$. Also let $\Delta T=T\left(S^{\prime}\right)-T(S)$. The following three cases must be considered.

Case 1. $C_{l_{2}}^{\mathrm{SPT}}<d_{i}<d_{j}$. In this case, we have $T\left(S^{\prime}\right)=T(S)=0$, so $\Delta T=0$.

Case $2 . d_{i} \leq C_{l_{2}}^{\mathrm{SPT}} \leq d_{j}$. We have $T(S)=\left(C_{l_{2}}^{\mathrm{SPT}}-d_{i}\right)^{2}$ and $T\left(S^{\prime}\right)=\left[\max \left(C_{l_{1}}^{\mathrm{SPT}}-\right.\right.$ $\left.\left.d_{i}, 0\right)\right]^{2}$. Consequently, $\Delta T=\left[\max \left(C_{l_{1}}^{\mathrm{SPT}}-d_{i}, 0\right)\right]^{2}-\left(C_{l_{2}}^{\mathrm{SPT}}-d_{i}\right)^{2}$. Therefore, we have $\Delta T \leq 0$, since $C_{l_{1}}^{\mathrm{SPT}}<C_{l_{2}}^{\mathrm{SPT}}$ and $d_{i} \leq C_{l_{2}}^{\mathrm{SPT}}$.

Case 3. $d_{i}<d_{j}<C_{l_{2}}^{\mathrm{SPT}}$. In this case, we have $T(S)=\left[\max \left(C_{l_{1}}^{\mathrm{SPT}}-d_{j}, 0\right)\right]^{2}+$ $\left(C_{l_{2}}^{\mathrm{SPT}}-d_{i}\right)^{2}$ and $T\left(S^{\prime}\right)=\left[\max \left(C_{l_{1}}^{\mathrm{SPT}}-d_{i}, 0\right)\right]^{2}+\left(C_{l_{2}}^{\mathrm{SPT}}-d_{j}\right)^{2}$. We then have $\Delta T=\left[\max \left(C_{l_{1}}^{\mathrm{SPT}}-d_{i}, 0\right)\right]^{2}+\left(C_{l_{2}}^{\mathrm{SPT}}-d_{j}\right)^{2}-\left[\max \left(C_{l_{1}}^{\mathrm{SPT}}-d_{j}, 0\right)\right]^{2}-$ $\left(C_{l_{2}}^{\mathrm{SPT}}-d_{i}\right)^{2}$. In order to simplify the analysis, this case can now be divided in three further subcases.

Case 3a. $C_{l_{1}}^{\mathrm{SPT}}<d_{i}<d_{j}<C_{l_{2}}^{\mathrm{SPT}}$. In this situation, we have $\Delta T=0+\left(C_{l_{2}}^{\mathrm{SPT}}-\right.$ $\left.d_{j}\right)^{2}-0-\left(C_{l_{2}}^{\mathrm{SPT}}-d_{i}\right)^{2}$. Therefore, we have $\Delta T<0$, since $d_{i}<d_{j}<C_{l_{2}}^{\mathrm{SPT}}$.

Case $3 \mathrm{~b} . d_{i} \leq C_{l_{1}}^{\mathrm{SPT}} \leq d_{j}<C_{l_{2}}^{\mathrm{SPT}}$. We now have $\Delta T=\left(C_{l_{1}}^{\mathrm{SPT}}-d_{i}\right)^{2}+\left(C_{l_{2}}^{\mathrm{SPT}}-d_{j}\right)^{2}-$ $0-\left(C_{l_{2}}^{\mathrm{SPT}}-d_{i}\right)^{2}$. Let $A=C_{l_{2}}^{\mathrm{SPT}}-d_{j}, B=d_{j}-C_{l_{1}}^{\mathrm{SPT}}$ and $C=C_{l_{1}}^{\mathrm{SPT}}-d_{i}$. We then have $\Delta T=C^{2}+A^{2}-(A+B+C)^{2}$. Therefore, $\Delta T \leq 0$, since with $A, B, C \geq 0$ we have $(A+B+C)^{2} \geq C^{2}+A^{2}$.

Case 3 c. $d_{i}<d_{j}<C_{l_{1}}^{\mathrm{SPT}}<C_{l_{2}}^{\mathrm{SPT}}$. We now have $\Delta \bar{T}=\left(C_{l_{1}}^{\mathrm{SPT}}-d_{i}\right)^{2}+\left(C_{l_{2}}^{\mathrm{SPT}}-d_{j}\right)^{2}-$ $\left(C_{l_{1}}^{\mathrm{SPT}}-d_{j}\right)^{2}-\left(C_{l_{2}}^{\mathrm{SPT}}-d_{i}\right)^{2}$. Let $A=C_{l_{2}}^{\mathrm{SPT}}-C_{l_{1}}^{\mathrm{SPT}}, B=C_{l_{1}}^{\mathrm{SPT}}-d_{j}$ and $C=d_{j}-d_{i}$. We then have $\Delta T=(B+C)^{2}+(A+B)^{2}-B^{2}-(A+B+C)^{2}$, with $A, B, C>0$. Expanding the squared terms and simplifying, we obtain $\Delta T=-2 A C<0$.

For all the situations considered, we have $\Delta E \leq 0$ and $\Delta T \leq 0$. Hence, scheduling jobs $i$ and $j$ in EDD order gives a lower value for $Z^{\prime}$. Consequently, we have $Z_{\mathrm{EDD}}^{\prime}=\min Z^{\prime}$ and $Z_{\mathrm{EDD}}^{\prime} \leq \min Z$, which concludes the proof.

\subsection{Special cases}

In this section, we present three special cases in which the lower bound is equal to the optimum objective function value. Let $C_{j}^{*}$ and $d_{j}^{*}$ denote the completion time 
and the due date, respectively, of the job scheduled in the $j$ th position in an optimal sequence. Also, let $Z^{*}=\sum_{j=1}^{n} \max \left(d_{j}^{*}-C_{j}^{*}, 0\right)+\sum_{j=1}^{n}\left[\max \left(C_{j}^{*}-d_{j}^{*}, 0\right)\right]^{2}$ denote the optimum objective function value. The following theorem provides three cases in which $Z_{\mathrm{EDD}}^{\prime}=Z^{*}$.

Theorem 2.2. $Z_{\mathrm{EDD}}^{\prime}=Z^{*}$, i.e. the lower bounding procedure gives the optimum objective function value, for each of the following cases:

Case 1. the EDD sequence is optimal and $p_{j}=p$, for all $j$;

Case 2. the EDD sequence is optimal, contains no tardy jobs, and is identical to the LPT sequence;

Case 3. the EDD sequence is optimal, contains no early jobs, and is identical to the SPT sequence.

Proof. For each of the three cases, we must show that the value provided by the lower bounding procedure is indeed equal to the objective function value of the optimal sequence.

Case 1. Given that the EDD sequence is optimal, we then have $d_{j}^{\mathrm{EDD}}=d_{j}^{*}$, for all $j$. Also, since the processing times are identical for all jobs, we have $C_{j}^{\mathrm{LPT}}=$ $C_{j}^{\mathrm{SPT}}=C_{j}^{*}$, for all $j$. Therefore, $Z_{\mathrm{EDD}}^{\prime}=\sum_{j=1}^{n} \max \left(d_{j}^{\mathrm{EDD}}-C_{j}^{\mathrm{LPT}}, 0\right)+$ $\sum_{j=1}^{n}\left[\max \left(C_{j}^{\mathrm{SPT}}-d_{j}^{\mathrm{EDD}}, 0\right)\right]^{2}=\sum_{j=1}^{n} \max \left(d_{j}^{*}-C_{j}^{*}, 0\right)+\sum_{j=1}^{n}\left[\max \left(C_{j}^{*}-\right.\right.$ $\left.\left.d_{j}^{*}, 0\right)\right]^{2}=Z^{*}$, so the lower bound is equal to the optimum objective function value.

Case 2. Since the EDD sequence is optimal, we again have $d_{j}^{\mathrm{EDD}}=d_{j}^{*}$, for all $j$. Furthermore, the EDD and LPT sequences are identical, so $C_{j}^{\mathrm{LPT}}=$ $C_{j}^{*}$. Given that the optimal sequence contains no tardy jobs, we then have $\sum_{j=1}^{n}\left[\max \left(C_{j}^{*}-d_{j}^{*}, 0\right)\right]^{2}=0$ and $Z^{*}=\sum_{j=1}^{n} \max \left(d_{j}^{*}-C_{j}^{*}, 0\right)=$ $\sum_{j=1}^{n} \max \left(d_{j}^{\mathrm{EDD}}-C_{j}^{\mathrm{LPT}}, 0\right)$. Also, we have $\sum_{j=1}^{n}\left[\max \left(C_{j}^{\mathrm{SPT}}-d_{j}^{\mathrm{EDD}}, 0\right)\right]^{2}=$ $\sum_{j=1}^{n}\left[\max \left(C_{j}^{\mathrm{SPT}}-d_{j}^{*}, 0\right)\right]^{2}=0$, since $\sum_{j=1}^{n}\left[\max \left(C_{j}^{*}-d_{j}^{*}, 0\right)\right]^{2}=0$ and $C_{j}^{\mathrm{SPT}} \leq C_{j}^{\mathrm{LPT}}=C_{j}^{*}$. Therefore, $Z_{\mathrm{EDD}}^{\prime}=\sum_{j=1}^{n} \max \left(d_{j}^{\mathrm{EDD}}-C_{j}^{\mathrm{LPT}}, 0\right)=Z^{*}$.

Case 3. The EDD sequence is once more optimal, so we have $d_{j}^{\mathrm{EDD}}=d_{j}^{*}$, for all $j$. Furthermore, since the EDD and SPT sequences are identical, we have $C_{j}^{\mathrm{SPT}}=C_{j}^{*}$. Given that the optimal sequence contains no early jobs, we then have $\sum_{j=1}^{n} \max \left(d_{j}^{*}-C_{j}^{*}, 0\right)=0$ and $Z^{*}=\sum_{j=1}^{n}\left[\max \left(C_{j}^{*}-d_{j}^{*}, 0\right)\right]^{2}=$ $\sum_{j=1}^{n}\left[\max \left(C_{j}^{\mathrm{SPT}}-d_{j}^{\mathrm{EDD}}, 0\right)\right]^{2}$. Also, we have $\sum_{j=1}^{n} \max \left(d_{j}^{\mathrm{EDD}}-C_{j}^{\mathrm{LPT}}, 0\right)=$ $\sum_{j=1}^{n} \max \left(d_{j}^{*}-C_{j}^{\mathrm{LPT}}, 0\right)=0$, since $\sum_{j=1}^{n} \max \left(d_{j}^{*}-C_{j}^{*}, 0\right)=0$ and $C_{j}^{\mathrm{LPT}} \geq$ $C_{j}^{\mathrm{SPT}}=C_{j}^{*}$. Therefore, $Z_{\mathrm{EDD}}^{\prime}=\sum_{j=1}^{n}\left[\max \left(C_{j}^{\mathrm{SPT}}-d_{j}^{\mathrm{EDD}}, 0\right)\right]^{2}=Z^{*}$, which concludes the proof.

\subsection{Applicability and extensions}

The lower bounding procedure creates a modified objective function, based on a relaxation of the completion times. For any specific sequence, the value of the modified objective function value is less than or equal to the value of the original function. 
The EDD ordering for the due dates is then shown to minimize the modified objective function, therefore providing a lower bound for the original objective. In this section, we present previous applications of this technique, and its generalization and applicability to other objective functions.

This lower bounding technique was previously used by Azizoglu et al. (1991) and Valente (2007) for two other early/tardy scheduling problems with no idle time. Azizoglu et al. (1991) applied this approach to derive a lower bound for the problem $\sum_{j=1}^{n}\left[(1-q) E_{j}+q T_{j}\right]$, with $0<q<1$, which is equivalent to the linear early/tardy problem with job-independent penalties $\sum_{j=1}^{n}\left(h E_{j}+w T_{j}\right)$. Valente (2007) instead considered the problem with job-dependent penalties and quadratic $\operatorname{costs} \sum_{j=1}^{n}\left(h_{j} E_{j}^{2}+w_{j} T_{j}^{2}\right)$. However, in order to derive a lower bound, the job penalties were first relaxed, in order to obtain a modified problem $\sum_{j=1}^{n}\left(h_{\min } E_{j}^{2}+\right.$ $w_{\min } T_{j}^{2}$, with $h_{\min }=\min \left\{h_{j} ; j=1, \ldots, n\right\}$ and $w_{\min }=\min \left\{w_{j} ; j=1, \ldots, n\right\}$. Therefore, the lower bounding technique was used to derive a lower bound for this modified problem with job-independent penalties. A somewhat similar approach was also used by Schaller (2004) for the $\sum_{j=1}^{n}\left(E_{j}+T_{j}^{2}\right)$ problem with inserted idle time. Therefore, and even though we focus on problems with no idle time, the lower bounding technique can also be extended to situations where the insertion of idle time is allowed.

The results given in this paper, together with those presented by Azizoglu et al. (1991) and Valente (2007), show that this technique provides a lower bound for each of the terms $\sum_{j=1}^{n} E_{j}, \sum_{j=1}^{n} E_{j}^{2}, \sum_{j=1}^{n} T_{j}$ and $\sum_{j=1}^{n} T_{j}^{2}$. Therefore, this approach can then be used to generate a lower bound for problems whose objective function is a convex combination of any of these four terms. This includes scheduling problems minimizing a sum of linear and/or quadratic earliness and tardiness, as well as problems with job-independent penalties.

The lower bounding technique is actually even more general since it can be used to obtain a lower bound for cubic and higher powers of the earliness and/or tardiness. Indeed, this approach yields a lower bound for any power $a \geq 1$. Let $E^{a}=\sum_{j=1}^{n} E_{j}^{a}$ and $T^{a}=\sum_{j=1}^{n} T_{j}^{a}$. Also, let $E_{\mathrm{EDD}}^{a}=\sum_{j=1}^{n}\left[\max \left(d_{j}^{\mathrm{EDD}}-C_{j}^{\mathrm{LPT}}, 0\right)\right]^{a}$ and $T_{\mathrm{EDD}}^{a}=\sum_{j=1}^{n}\left[\max \left(C_{j}^{\mathrm{SPT}}-d_{j}^{\mathrm{EDD}}, 0\right)\right]^{a}$. Therefore, in the problem considered in this paper we have $Z=E^{1}+T^{2}$ and $Z_{\mathrm{EDD}}^{\prime}=E_{\mathrm{EDD}}^{1}+T_{\mathrm{EDD}}^{2}$. The following theorem shows that the lower bounding technique can be generalized to all powers $a \geq 1$.

Theorem 2.3. $E_{\mathrm{EDD}}^{a} \leq \min E^{a}$ and $T_{\mathrm{EDD}}^{a} \leq \min T^{a}$ for $a \geq 1$, i.e. $E_{\mathrm{EDD}}^{a}$ and $T_{\mathrm{EDD}}^{a}$ provide a lower bound for the optimal objective function value of the $\sum_{j=1}^{n} E_{j}^{a}$ and $\sum_{j=1}^{n} T_{j}^{a}$ functions, respectively.

Proof. The results previously given in this paper, as well as those developed by Azizoglu et al. (1991) and Valente (2007), have already showed that the theorem is valid for $a=1,2$. A proof similar to those previously presented for these linear and quadratic functions could also be used to show that the same applies to other values of $a$. For brevity, however, we will remark that all the cases and subcases that would have to be considered in the general proof can be classified into one of 
three categories. In the remainder of this proof, we first illustrate these categories with an example from the proof we previously presented for Theorem 2.1. More specifically, we will use as examples some of the cases considered for the quadratic tardiness component of our objective function. Then, we show that the proof given for that case is also valid for a general power $a \geq 1$.

Category 2.1. In this category, the proof of the case is obvious from the relations between the due dates and the completion times. For example, consider the proof of Case 2. In this case, we have $d_{i} \leq C_{l_{2}}^{\mathrm{SPT}} \leq d_{j}$, and the proof for $a=2$ requires that $\left[\max \left(C_{l_{1}}^{\mathrm{SPT}}-d_{i}, 0\right)\right]^{2}-\left(C_{l_{2}}^{\mathrm{SPT}}-d_{i}\right)^{2} \leq 0$. Therefore, the proof for a general $a \geq 1$ would require that $\left[\max \left(C_{l_{1}}^{\mathrm{SPT}}-d_{i}, 0\right)\right]^{a}-\left(C_{l_{2}}^{\mathrm{SPT}}-d_{i}\right)^{a} \leq 0$. This inequality does hold, since $C_{l_{1}}^{\mathrm{SPT}}<C_{l_{2}}^{\mathrm{SPT}}$ and $d_{i} \leq C_{l_{2}}^{\mathrm{SPT}}$.

Category 2.2. In this category, the case can be proved by showing that $C^{a}+A^{a}-$ $(A+B+C)^{a} \leq 0$. Case $3 \mathrm{~b}$ gives an example of this category, since its proof requires that $C^{2}+A^{2}-(A+B+C)^{2} \leq 0$. Since we have $A, B, C \geq 0$, it is then clear that we have $C^{a}+A^{a}-(A+B+C)^{a} \leq 0$.

Category 2.3. In this final category, the case can be proved by showing that $(B+C)^{a}+(A+B)^{a}-B^{a}-(A+B+C)^{a} \leq 0$. Case $3 c$ provides an example of this category. We first recall that $A, B, C \geq 0$. Newton's binomial theorem can then be used to expand the expression $(B+C)^{a}+(A+B)^{a}-B^{a}-(A+B+C)^{a}$. It is then clear, from the expanded expression, that we have $(B+C)^{a}+(A+B)^{a}-B^{a}-(A+B+C)^{a} \leq$ 0 . For the sake of brevity, we omit the details.

The lower bounding technique, however, cannot be used to obtain a lower bound for some other earliness and/or tardiness cost functions. In fact, simple counterexamples show that the approach fails for objective functions with powers $0<a<1$. Also, as previously mentioned, the technique is appropriate for objective functions that minimize a simple sum of earliness and tardiness costs, as well as for problems with job-independent penalties. However, this approach is not suited to the minimization of objective functions with job-dependent penalties, such as $\sum_{j=1}^{n} h_{j} E_{j}^{2}$ or $\sum_{j=1}^{n} w_{j} T_{j}$. As described above, Valente (2007) considered the problem with job-dependent penalties $\sum_{j=1}^{n}\left(h_{j} E_{j}^{2}+w_{j} T_{j}^{2}\right)$. Nevertheless, a relaxation of the job penalties was first performed, and the lower bounding technique was then applied to a modified problem with job-independent penalties.

\section{Branch-and-Bound Procedure}

In this section, we discuss the implementation details of the branch-and-bound algorithm. We use a forward-sequencing branching rule, where a node at level $l$ of the search tree corresponds to a sequence with $l$ jobs fixed in the first $l$ positions. The depth-first strategy is used to search the tree, and ties are broken by selecting the node with the smallest value of the associated partial schedule cost plus the associated lower bound for the unscheduled jobs. The tie-breaking strategy therefore 
chooses the node that seems most promising, i.e. the node that will most likely lead to a complete schedule with a lower objective function value.

The initial upper bound on the optimum schedule cost is calculated using the EQTP-EXP dispatching rule. The EQTP-EXP heuristic calculates a priority index for each remaining job every time the machine becomes available, and the job with the highest priority is then selected to be processed next. Let $I_{j}(t)$ denote the priority index of job $J_{j}$ at time $t$. The EQTP-EXP dispatching rule then uses the following priority index $I_{j}(t)$ :

$$
I_{j}(t)= \begin{cases}\left(1 / p_{j}\right)\left[\bar{p}+2\left(t+p_{j}-d_{j}\right)\right] & \text { if } s_{j} \leq 0 \\ \left(\bar{p} / p_{j}\right) \exp \left[-(\bar{p}+1) s_{j} / k \bar{p}\right] & \text { if } 0<s_{j}<[\bar{p} /(\bar{p}+1)] k \bar{p} \\ \left(1 / p_{j}\right)^{-2}\left[\left(\bar{p} / p_{j}\right)-\left(1 / p_{j}\right)(\bar{p}+1) s_{j} / k \bar{p}\right]^{3} & \text { if }[\bar{p} /(\bar{p}+1)] k \bar{p} \leq s_{j}<k \bar{p} \\ -\left(1 / p_{j}\right) & \text { otherwise, }\end{cases}
$$

where $s_{j}=d_{j}-t-p_{j}$ is the slack of job $J_{j}, \bar{p}$ is the average processing time of the remaining unscheduled jobs and $k$ is a lookahead parameter. The EQTP_EXP procedure provided the best results among all the heuristics analyzed in Valente (2006). The upper bound value is then updated whenever a feasible schedule with a lower cost is found during the branching process.

Two fathoming tests are used to reduce the number of nodes in the search tree. In the first test, an insertion-based procedure is used to eliminate dominated nodes. This procedure inserts the job most recently added to the node's partial sequence before a certain number of the previously scheduled jobs. If an improving sequence is found, the node is then fathomed.

We considered six alternative versions of the branch-and-bound procedure. These versions differ only in the number (denoted by INS) of previously scheduled jobs that are considered in the insertion fathoming test. In the version INS = single, only one insertion is performed. In the version INS $=0.10$ (respectively, 0.25, 0.50, 0.75 and 1.00), on the other hand, the number of insertions that are considered is equal to $10 \%$ (respectively, $25 \%, 50 \%, 75 \%$ and $100 \%$ ) of the number of previously scheduled jobs.

When the node is not eliminated by the previous test, a lower bound is then calculated for the unscheduled jobs. If the lower bound plus the cost of the associated partial schedule is larger than or equal to the current upper bound, the node is discarded.

\section{Computational Results}

In this section, we first describe the set of test problems used in the computational experiments. Then, we analyze the performance of the lower bounding procedure. Finally, we present computational results for the branch-and-bound algorithms. Throughout this section, and in order to avoid excessively large tables, we will sometimes present results only for some representative cases. 


\subsection{Experimental design}

The computational tests were performed on a set of problems with $7,10,12,15,17$ and 20 jobs. These problems were randomly generated as follows. For each job $J_{j}$, an integer processing time $p_{j}$ was generated from one of the two uniform distributions $[1,10]$ and $[1,100]$, in order to obtain a low $(\mathrm{L})$ and a high $(\mathrm{H})$ range, respectively, for the processing time values. For each job $J_{j}$, an integer due date $d_{j}$ is generated from the uniform distribution $[P(1-T-R / 2), P(1-T+R / 2)]$, where $P$ is the sum of the processing times of all jobs, $T$ is the tardiness factor, set at $0.0,0.2,0.4$, $0.6,0.8$ and 1.0, and $R$ is the range of due dates, set at $0.2,0.4,0.6$ and 0.8 .

For each combination of problem size $n$, processing time range (rng), $T$ and $R, 50$ instances were randomly generated. Therefore, a total of 1200 instances was generated for each combination of problem size and processing time range. All the algorithms were coded in Visual $\mathrm{C}++6.0$, and executed on a Pentium $\mathrm{IV}-2.8 \mathrm{GHz}$ personal computer.

\subsection{Lower bound results}

In this section, we analyze the performance of the lower bound. In Table 1, we present the average of the relative deviations from the optimum, calculated as $(O-L B) / O \times 100$, where $O$ and $L B$ represent the optimum objective function value and the initial lower bound value (i.e. the lower bound at the root node), respectively. From the results given in this table, we can see that the lower bound value is, on average, $25 \%$ to $30 \%$ below the optimum. Also, the performance of the lower bounding procedure is somewhat better when the range of processing times is low.

The effect of the $T$ and $R$ parameters on the relative deviation from the optimum is given in Table 2. The lower bound performs better when $T=0.0$ or $T=1.0$. Therefore, the lower bound value is closer to the optimum when nearly all jobs are early $(T=0.0)$ or tardy $(T=1.0)$. The performance of the lower bound is particularly good for instances where most jobs are early. In fact, the lower bound is usually quite close to the optimum for instances with $T=0.0$, especially when the range of the due dates $R$ is low.

The lower bound's relative deviation from the optimum then increases as the tardiness factor $T$ approaches its intermediate values. Therefore, the performance of

Table 1. Relative deviation from the optimum.

\begin{tabular}{rcc}
\hline$n$ & Low rng & High rng \\
\hline 7 & 24.63 & 25.01 \\
10 & 26.41 & 27.58 \\
12 & 26.87 & 27.64 \\
15 & 27.14 & 28.97 \\
17 & 26.92 & 28.57 \\
20 & 27.58 & 29.22 \\
\hline
\end{tabular}


Table 2. Effect of the tardiness factor and the range of due dates on the relative deviation from the optimum.

\begin{tabular}{|c|c|c|c|c|c|c|c|c|c|}
\hline \multirow[t]{2}{*}{$n$} & \multirow[t]{2}{*}{$T$} & \multicolumn{4}{|c|}{ Low rng } & \multicolumn{4}{|c|}{ High rng } \\
\hline & & $R=0.2$ & $R=0.4$ & $R=0.6$ & $R=0.8$ & $R=0.2$ & $R=0.4$ & $R=0.6$ & $R=0.8$ \\
\hline \multirow[t]{6}{*}{10} & 0.0 & 1.47 & 2.62 & 5.39 & 7.03 & 1.99 & 4.62 & 7.41 & 13.77 \\
\hline & 0.2 & 18.33 & 24.46 & 28.82 & 37.44 & 15.38 & 32.93 & 29.65 & 41.77 \\
\hline & 0.4 & 15.63 & 33.97 & 44.08 & 74.60 & 19.43 & 30.68 & 41.10 & 70.31 \\
\hline & 0.6 & 17.74 & 29.40 & 42.60 & 54.73 & 18.52 & 29.28 & 45.83 & 53.36 \\
\hline & 0.8 & 16.11 & 27.11 & 31.52 & 39.54 & 12.19 & 27.96 & 38.00 & 45.85 \\
\hline & 1.0 & 10.47 & 19.03 & 23.89 & 27.77 & 10.65 & 17.73 & 24.76 & 28.84 \\
\hline \multirow[t]{6}{*}{15} & 0.0 & 0.90 & 2.33 & 3.85 & 5.23 & 1.55 & 2.68 & 5.31 & 6.79 \\
\hline & 0.2 & 17.96 & 22.12 & 30.91 & 26.81 & 18.36 & 32.59 & 32.84 & 40.54 \\
\hline & 0.4 & 20.02 & 34.44 & 51.57 & 77.10 & 22.33 & 33.33 & 42.23 & 71.11 \\
\hline & 0.6 & 17.44 & 29.84 & 44.66 & 51.79 & 21.68 & 32.25 & 45.13 & 61.56 \\
\hline & 0.8 & 16.33 & 26.89 & 39.39 & 46.17 & 17.19 & 27.97 & 38.50 & 50.32 \\
\hline & 1.0 & 11.13 & 18.68 & 26.53 & 29.28 & 11.23 & 21.63 & 27.04 & 31.06 \\
\hline \multirow[t]{6}{*}{20} & 0.0 & 0.75 & 2.15 & 3.86 & 5.44 & 0.99 & 2.92 & 5.63 & 6.92 \\
\hline & 0.2 & 16.89 & 20.39 & 24.19 & 30.12 & 21.24 & 30.38 & 29.10 & 30.43 \\
\hline & 0.4 & 23.10 & 32.10 & 50.08 & 72.58 & 21.52 & 34.02 & 46.71 & 72.54 \\
\hline & 0.6 & 20.83 & 34.37 & 42.61 & 59.09 & 20.44 & 31.56 & 47.97 & 64.53 \\
\hline & 0.8 & 16.34 & 26.99 & 39.04 & 47.05 & 16.99 & 29.39 & 42.33 & 50.55 \\
\hline & 1.0 & 12.12 & 20.75 & 27.59 & 33.43 & 11.52 & 22.72 & 28.05 & 32.72 \\
\hline
\end{tabular}

the lower bounding procedure deteriorates when there is a greater balance between the number of early and tardy jobs.

The range of due dates parameter also has a noticeable impact on the effectiveness of the lower bound. In fact, the relative deviation from the optimum becomes higher as $R$ increases. Consequently, the lower bound performs better when the due dates are quite close, and its performance deteriorates as the due dates become more widely spread.

\subsection{Branch-and-bound results}

The results for the branch-and-bound algorithms will be presented in this section. In Table 3, we give the branch-and-bound average computation times, in seconds. The branch-and-bound procedures are capable of solving, within reasonable computation times, problems with up to 20 jobs. The range of the processing times has a significant effect on the runtimes since the branch-and-bound procedures are much faster for instances with a high processing time range.

For the smaller instances, the computation times are similar for the various INS values. The difference in the runtimes, however, becomes much clearer for the larger instances with 17 and 20 jobs. For these instance sizes, the branchand-bound procedures with INS $=$ single or INS $=0.10$ require a substantially higher computation time. The runtimes for the remaining INS values are somewhat close even though the best performance is given by the INS $=0.50$ and INS $=$ 0.75 algorithms. When the INS parameter is higher, the insertion fathoming test 
Table 3. Branch-and-bound runtimes (in seconds).

\begin{tabular}{|c|c|c|c|c|c|c|c|}
\hline \multirow[t]{2}{*}{ rng } & \multirow[t]{2}{*}{$n$} & \multicolumn{6}{|c|}{ INS } \\
\hline & & single & 0.10 & 0.25 & 0.50 & 0.75 & 1.00 \\
\hline \multirow[t]{6}{*}{$\mathrm{L}$} & 7 & 0.000 & 0.000 & 0.000 & 0.001 & 0.001 & 0.001 \\
\hline & 10 & 0.007 & 0.005 & 0.004 & 0.007 & 0.007 & 0.007 \\
\hline & 12 & 0.042 & 0.029 & 0.027 & 0.039 & 0.038 & 0.041 \\
\hline & 15 & 0.779 & 0.748 & 0.646 & 0.641 & 0.632 & 0.660 \\
\hline & 17 & 7.534 & 7.638 & 5.967 & 5.370 & 5.344 & 5.503 \\
\hline & 20 & 266.843 & 253.358 & 180.464 & 159.420 & 157.162 & 159.991 \\
\hline \multirow[t]{6}{*}{$\mathrm{H}$} & 7 & 0.001 & 0.001 & 0.001 & 0.001 & 0.001 & 0.001 \\
\hline & 10 & 0.006 & 0.007 & 0.006 & 0.006 & 0.006 & 0.006 \\
\hline & 12 & 0.031 & 0.032 & 0.031 & 0.030 & 0.031 & 0.031 \\
\hline & 15 & 0.551 & 0.558 & 0.474 & 0.429 & 0.432 & 0.446 \\
\hline & 17 & 3.569 & 3.625 & 2.884 & 2.629 & 2.670 & 2.755 \\
\hline & 20 & 83.140 & 80.036 & 52.758 & 48.010 & 49.241 & 50.761 \\
\hline
\end{tabular}

will usually eliminate more nodes, but it will also require additional computation time. Therefore, increasing the number of insertions has two opposite effects on the efficiency of the branch-and-bound algorithm. The best results were then achieved when the number of insertions is relatively high (INS between 0.50 and 0.75 ), but still below its maximum possible value.

In Table 4, we present several additional statistics for the computation times, namely the minimum ( $\min$ ) and maximum ( $\max$ ) values, the coefficient of variation (cov), and the percentiles 25, 50, 75, 95 and 99 (p25, p50, p75, p95 and p99, respectively) of the distribution of the runtimes. The results in Table 4 once again show that the procedures with INS $=$ single or INS $=0.10$ require higher computation times. The remaining values of the INS parameter (particularly the values 0.50 and 0.75) provide superior results and are also significantly more consistent, since the variability in their runtimes is lower, as indicated by the cov values.

The improvement in performance provided by the higher values of the parameter INS becomes larger as the instance difficulty increases. For the easier instances (min, p25 and p50), which require low computation times, the runtimes are close for all INS values. As the instance difficulty, and correspondingly the runtime, increase, the branch-and-bound procedures with INS equal or greater than 0.25 become increasingly more efficient, since the increase in the runtime is much slower for these procedures. For the most difficult instances (p95, p99 and max), the computation times are substantially lower for the algorithms with a INS value of at least 0.25 .

The effect of the $T$ and $R$ parameters on the computation times for instances with 20 jobs is given in Table 5. The results presented in this table show that the tardiness factor $T$ has a clear and significant effect on the runtimes. Indeed, the branch-and-bound procedures are quite fast when $T=0.0$ or $T=1.0$. The runtimes increase significantly, however, as $T$ approaches its intermediate values. Therefore, 
Table 4. Branch-and-bound runtimes statistics.

\begin{tabular}{|c|c|c|c|c|c|c|c|c|c|c|}
\hline rng & $n$ & INS & $\min$ & p25 & p50 & p75 & p95 & p99 & $\max$ & $\mathrm{cov}$ \\
\hline \multirow[t]{12}{*}{$\mathrm{L}$} & 15 & single & 0.000 & 0.063 & 0.187 & 0.735 & 3.578 & 8.227 & 16.375 & 208.0 \\
\hline & & 0.10 & 0.000 & 0.0 & & & & & 15.674 & 206.9 \\
\hline & & 0.25 & 0.000 & 0.061 & 170 & 629 & 3.044 & 6.399 & 11.729 & 193.0 \\
\hline & & 0.50 & 0.000 & 0.078 & 187 & .594 & 2.774 & 6.187 & 13.516 & 192.1 \\
\hline & & 0.75 & 0.000 & 0.07 & & & & & & 191.1 \\
\hline & & 1.00 & 0.000 & 0.078 & 0.203 & 0.617 & 2.937 & 6.438 & 14.672 & 190.7 \\
\hline & 20 & single & 0.000 & 2.672 & 10.196 & 96.945 & 1110.895 & 4408.280 & 22466.100 & 439.4 \\
\hline & & 0.10 & 0.000 & 2.632 & 10.343 & 99.7 & 1084.160 & 4128. & 300 & 418.6 \\
\hline & & 0.25 & 0.000 & 2.562 & 8.693 & 76.031 & 770.061 & 3033.090 & 11926. & 402.0 \\
\hline & & 0.50 & 0.000 & 2.687 & 8.555 & 68.478 & 669.931 & 2478.330 & 360. & 388.0 \\
\hline & & 0.75 & 0.000 & 2.812 & 9.166 & 66.734 & 703.789 & 2434.775 & 8724.430 & 372.2 \\
\hline & & 1.00 & 0.000 & 2.867 & 9.352 & 69.101 & 727.679 & 2472.930 & 8913.630 & 368.2 \\
\hline \multirow[t]{12}{*}{$\mathrm{H}$} & 15 & single & 0.000 & 0.047 & 0.172 & 0.547 & 2.429 & 4.83 & & 193.6 \\
\hline & & 0.10 & 0.000 & 0.047 & 0.172 & & 2.470 & 5.0 & & 194.6 \\
\hline & & 0.25 & 0.000 & 0.047 & 0.172 & 0.516 & 1.992 & & 12.609 & 181.0 \\
\hline & & 0.50 & 0.000 & 0.047 & 0.157 & 0.469 & 1.758 & 3.406 & 12.516 & 181.1 \\
\hline & & 0.75 & 0.000 & 0.047 & 0.172 & 0.484 & 1.688 & 3.493 & & 182.3 \\
\hline & & 1.00 & 0.000 & 0.062 & 0.172 & 0.500 & 1.758 & 3.626 & 13.328 & 182.4 \\
\hline & 20 & single & 0.000 & 1.188 & 7.734 & 53.453 & 372.126 & 1410.945 & 3184.880 & 305.5 \\
\hline & & 0.10 & 0.000 & 1.196 & 7.742 & 52.624 & 384.672 & 1392.995 & 3135.770 & 297.0 \\
\hline & & 0.25 & 0.000 & 1.188 & 6.336 & 39.687 & 223.420 & 841.878 & 2327.460 & 287.2 \\
\hline & & 0.50 & 0.000 & 1.219 & 5.805 & 36.281 & 221.044 & 783.361 & 1779.420 & 287.7 \\
\hline & & 0.75 & 0.000 & 1.281 & 5.898 & 36.562 & 224.779 & 811.123 & 1921.410 & 292.4 \\
\hline & & 1.00 & 0.000 & 1.328 & 6.000 & 37.874 & 230.710 & 836.464 & 1980.080 & 292.8 \\
\hline
\end{tabular}

Table 5. Branch-and-bound runtimes (in seconds) for instances with 20 jobs.

\begin{tabular}{|c|c|c|c|c|c|c|c|c|c|}
\hline \multirow[t]{2}{*}{ INS } & \multirow[t]{2}{*}{$T$} & \multicolumn{4}{|c|}{ Low rng } & \multicolumn{4}{|c|}{ High rng } \\
\hline & & $R=0.2$ & $R=0.4$ & $R=0.6$ & $R=0.8$ & $R=0.2$ & $R=0.4$ & $R=0.6$ & $R=0.8$ \\
\hline \multirow{6}{*}{ single } & 0.0 & 1.379 & 2.968 & 7.181 & 7.834 & 0.046 & 0.178 & 0.469 & 0.667 \\
\hline & 0.2 & 144.480 & 54.097 & 14.172 & 10.206 & 19.489 & 10.494 & 2.346 & 2.020 \\
\hline & 0.4 & 1675.648 & 602.005 & 257.300 & 108.419 & 63.151 & 157.273 & 206.859 & 63.196 \\
\hline & 0.6 & 1815.821 & 1149.630 & 137.527 & 39.781 & 447.947 & 370.219 & 196.340 & 62.631 \\
\hline & 0.8 & 266.418 & 45.660 & 17.196 & 16.536 & 232.023 & 81.376 & 34.557 & 12.363 \\
\hline & 1.0 & 3.905 & 7.627 & 10.898 & 7.535 & 3.268 & 12.396 & 9.107 & 6.941 \\
\hline \multirow[t]{6}{*}{0.10} & 0.0 & 1.626 & 2.966 & 7.384 & 7.936 & 0.048 & 0.181 & 0.475 & 0.671 \\
\hline & 0.2 & 143.606 & 53.186 & 14.338 & 10.307 & 19.284 & 10.128 & 2.336 & 2.020 \\
\hline & 0.4 & 1509.562 & 588.645 & 258.094 & 108.411 & 60.043 & 148.263 & 191.502 & 62.291 \\
\hline & 0.6 & 1706.668 & 1110.604 & 135.878 & 39.939 & 416.307 & 358.686 & 192.187 & 62.343 \\
\hline & 0.8 & 271.429 & 46.051 & 17.221 & 16.463 & 235.718 & 80.196 & 33.937 & 12.219 \\
\hline & 1.0 & 3.950 & 7.774 & 10.977 & 7.573 & 3.275 & 12.588 & 9.236 & 6.935 \\
\hline \multirow[t]{6}{*}{0.25} & 0.0 & 1.450 & 2.707 & 7.557 & 8.120 & 0.047 & 0.184 & 0.495 & 0.679 \\
\hline & 0.2 & 145.280 & 50.477 & 14.465 & 10.295 & 19.351 & 9.500 & 2.198 & 1.984 \\
\hline & 0.4 & 1160.361 & 499.310 & 205.845 & 90.866 & 54.412 & 118.689 & 158.936 & 50.635 \\
\hline & 0.6 & 1047.229 & 740.272 & 88.983 & 32.016 & 263.000 & 224.767 & 117.488 & 39.794 \\
\hline & 0.8 & 152.211 & 26.654 & 11.991 & 11.869 & 111.886 & 45.393 & 16.974 & 8.455 \\
\hline & 1.0 & 3.803 & 5.856 & 6.976 & 6.541 & 3.018 & 7.893 & 5.755 & 4.659 \\
\hline
\end{tabular}


Table 5. (Continued)

\begin{tabular}{|c|c|c|c|c|c|c|c|c|c|}
\hline \multirow[t]{2}{*}{ INS } & \multirow[t]{2}{*}{$T$} & \multicolumn{4}{|c|}{ Low rng } & \multicolumn{4}{|c|}{ High rng } \\
\hline & & $R=0.2$ & $R=0.4$ & $R=0.6$ & $R=0.8$ & $R=0.2$ & $R=0.4$ & $R=0.6$ & $R=0.8$ \\
\hline \multirow[t]{6}{*}{0.50} & 0.0 & 2.317 & 3.811 & 8.472 & 8.709 & 0.048 & 0.188 & 0.499 & 0.702 \\
\hline & 0.2 & 158.499 & 50.847 & 15.077 & 10.809 & 19.619 & 9.753 & 2.241 & 2.039 \\
\hline & 0.4 & 1045.781 & 486.379 & 187.088 & 77.886 & 54.738 & 117.973 & 160.013 & 47.774 \\
\hline & 0.6 & 876.977 & 617.390 & 74.817 & 26.632 & 253.962 & 201.571 & 100.069 & 30.670 \\
\hline & 0.8 & 116.064 & 19.067 & 9.257 & 9.953 & 80.746 & 34.797 & 11.159 & 6.872 \\
\hline & 1.0 & 3.343 & 4.807 & 5.969 & 6.138 & 2.223 & 5.632 & 4.641 & 4.303 \\
\hline \multirow[t]{6}{*}{0.75} & 0.0 & 2.575 & 4.188 & 9.186 & 9.340 & 0.054 & 0.202 & 0.530 & 0.743 \\
\hline & 0.2 & 176.349 & 53.486 & 15.972 & 11.474 & 20.361 & 10.243 & 2.357 & 2.154 \\
\hline & 0.4 & 1040.547 & 502.557 & 190.703 & 77.972 & 56.982 & 123.203 & 169.323 & 49.786 \\
\hline & 0.6 & 845.262 & 573.299 & 73.784 & 23.989 & 262.623 & 211.588 & 101.038 & 28.623 \\
\hline & 0.8 & 103.792 & 17.531 & 9.053 & 9.396 & 75.960 & 30.699 & 11.193 & 6.868 \\
\hline & 1.0 & 3.562 & 5.101 & 6.247 & 6.532 & 2.292 & 5.634 & 4.854 & 4.470 \\
\hline \multirow[t]{6}{*}{1.00} & 0.0 & 1.847 & 3.741 & 9.361 & 9.710 & 0.057 & 0.208 & 0.559 & 0.778 \\
\hline & 0.2 & 184.757 & 56.006 & 16.632 & 11.913 & 20.876 & 10.627 & 2.432 & 2.219 \\
\hline & 0.4 & 1067.915 & 518.150 & 196.666 & 79.596 & 59.031 & 127.459 & 175.826 & 51.451 \\
\hline & 0.6 & 852.607 & 567.286 & 74.987 & 24.976 & 269.587 & 217.613 & 104.450 & 29.956 \\
\hline & 0.8 & 103.458 & 18.285 & 9.472 & 9.747 & 76.624 & 31.253 & 11.803 & 7.235 \\
\hline & 1.0 & 3.821 & 5.406 & 6.556 & 6.895 & 2.437 & 5.953 & 5.120 & 4.716 \\
\hline
\end{tabular}

the problem becomes harder to solve when there is a greater balance between the number of early and tardy jobs.

In Table 6 , we present the average number of nodes generated by the branchand-bound algorithm (NG), as well as the average percentage of these nodes that were eliminated by the two fathoming tests (\%EL). We also provide some data on the relative importance of these tests, namely the average percentage of nodes fathomed by the lower bound test (\%LB) and the insertion-based procedure (\%INS). The number of nodes generated is higher for the instances with a low processing time range. Additionally, this number decreases with the value of the INS parameter. These results are in line with the computation times previously presented in Table 3.

The proportion of nodes eliminated by the fathoming tests increases with the instance size, and is marginally higher for instances with a high processing time range. Also, the percentage of eliminated nodes increases very slightly with the INS parameter. The relative importance of the lower bound fathoming test is lower for the instances with a high processing time range, and decreases with the instance size, while the proportion of nodes eliminated by the insertion procedure correspondingly increases. The relative importance of the insertion-based test also increases with the INS parameter. This is to be expected since more insertions are performed for the higher INS values.

In Table 7, we present the effect of the $T$ and $R$ parameters on the average number of nodes generated and the percentage of nodes eliminated by the lower 
Table 6. Average number of nodes and relative importance of the fathoming tests.

\begin{tabular}{|c|c|c|c|c|c|c|c|c|c|}
\hline \multirow[t]{2}{*}{$n$} & \multirow[t]{2}{*}{ INS } & \multicolumn{4}{|c|}{ Low rng } & \multicolumn{4}{|c|}{ High rng } \\
\hline & & NG & $\% \mathrm{EL}$ & $\%$ LB & \%INS & NG & $\% \mathrm{EL}$ & $\% \mathrm{LB}$ & \%INS \\
\hline \multirow[t]{6}{*}{10} & single & 1683 & 83.08 & 49.65 & 50.35 & 1635 & 83.25 & 44.29 & 55.71 \\
\hline & 0.10 & 1683 & 83.08 & 49.65 & 50.35 & 1635 & 83.25 & 44.29 & 55.71 \\
\hline & 0.25 & 1667 & 83.14 & 49.01 & 50.99 & 1620 & 83.31 & 43.69 & 56.31 \\
\hline & 0.50 & 1596 & 83.27 & 47.56 & 52.44 & 1554 & 83.44 & 42.39 & 57.61 \\
\hline & 0.75 & 1555 & 83.32 & 46.89 & 53.11 & 1516 & 83.50 & 41.81 & 58.19 \\
\hline & 1.00 & 1552 & 83.33 & 46.83 & 53.17 & 1515 & 83.50 & 41.78 & 58.22 \\
\hline \multirow[t]{6}{*}{15} & single & 202219 & 87.78 & 43.61 & 56.39 & 143699 & 88.19 & 38.12 & 61.88 \\
\hline & 0.10 & 202171 & 87.78 & 43.58 & 56.42 & 143633 & 88.20 & 38.08 & 61.92 \\
\hline & 0.25 & 176435 & 87.96 & 41.44 & 58.56 & 122348 & 88.42 & 35.73 & 64.27 \\
\hline & 0.50 & 157738 & 88.07 & 40.04 & 59.96 & 107782 & 88.52 & 34.47 & 65.53 \\
\hline & 0.75 & 151430 & 88.09 & 39.60 & 60.40 & 103124 & 88.55 & 34.12 & 65.88 \\
\hline & 1.00 & 150659 & 88.09 & 39.54 & 60.46 & 102803 & 88.55 & 34.08 & 65.92 \\
\hline \multirow[t]{6}{*}{20} & single & 64614975 & 90.27 & 40.55 & 59.45 & 19979272 & 90.76 & 35.24 & 64.76 \\
\hline & 0.10 & 60581125 & 90.32 & 39.90 & 60.10 & 19039709 & 90.81 & 34.59 & 65.41 \\
\hline & 0.25 & 43370259 & 90.51 & 37.13 & 62.87 & 12725387 & 91.05 & 31.64 & 68.36 \\
\hline & 0.50 & 36809014 & 90.56 & 36.05 & 63.95 & 10986024 & 91.11 & 30.72 & 69.28 \\
\hline & 0.75 & 34645609 & 90.58 & 35.74 & 64.26 & 10590399 & 91.12 & 30.52 & 69.48 \\
\hline & 1.00 & 34182075 & 90.58 & 35.69 & 64.31 & 10550631 & 91.12 & 30.50 & 69.50 \\
\hline
\end{tabular}

Table 7. Nodes generated and importance of lower bound test for INS $=0.50$.

\begin{tabular}{|c|c|c|c|c|c|c|c|c|c|c|}
\hline \multirow[t]{2}{*}{ rng } & \multirow[t]{2}{*}{$n$} & \multirow[t]{2}{*}{$T$} & \multicolumn{2}{|c|}{$R=0.2$} & \multicolumn{2}{|c|}{$R=0.4$} & \multicolumn{2}{|c|}{$R=0.6$} & \multicolumn{2}{|c|}{$R=0.8$} \\
\hline & & & NG & \%LB & NG & $\%$ LB & NG & $\% \mathrm{LB}$ & NG & $\% \mathrm{LB}$ \\
\hline \multirow[t]{18}{*}{$\mathrm{L}$} & 10 & 0.0 & 215 & 87.49 & 383 & 84.78 & 533 & 78.35 & 603 & 75.06 \\
\hline & & 0.2 & 1654 & 43.57 & 1287 & 53.34 & 1101 & 56.82 & 958 & 59.17 \\
\hline & & 0.4 & 3683 & 27.19 & 3263 & 32.10 & 2318 & 39.38 & 1091 & 43.37 \\
\hline & & 0.6 & 3545 & 38.60 & 2931 & 38.10 & 2390 & 35.81 & 1479 & 37.27 \\
\hline & & 0.8 & 2136 & 43.84 & 1688 & 36.88 & 1217 & 41.45 & 1124 & 39.54 \\
\hline & & 1.0 & 1018 & 53.57 & 1128 & 43.61 & 1271 & 41.61 & 1294 & 37.67 \\
\hline & 15 & 0.0 & 15432 & 84.81 & 17071 & 78.77 & 20627 & 75.11 & 17329 & 69.79 \\
\hline & & 0.2 & 347280 & 29.02 & 70254 & 45.58 & 70331 & 45.82 & 52411 & 55.71 \\
\hline & & 0.4 & 565606 & 20.75 & 515549 & 26.11 & 263003 & 33.89 & 117987 & 37.20 \\
\hline & & 0.6 & 457500 & 36.42 & 423822 & 32.64 & 262144 & 28.06 & 56996 & 29.07 \\
\hline & & 0.8 & 134916 & 36.42 & 113028 & 29.74 & 75948 & 24.28 & 53906 & 24.43 \\
\hline & & 1.0 & 22164 & 42.18 & 37504 & 31.97 & 39427 & 29.89 & 35472 & 29.84 \\
\hline & 20 & 0.0 & 440300 & 82.77 & 770813 & 75.06 & 1948321 & 68.64 & 2003348 & 61.48 \\
\hline & & 0.2 & 48999699 & 23.65 & 13395740 & 44.10 & 4097621 & 46.52 & 2565839 & 45.50 \\
\hline & & 0.4 & 258166460 & 23.26 & 111282106 & 26.91 & 44457661 & 30.37 & 18573874 & 38.23 \\
\hline & & 0.6 & 169508421 & 34.51 & 145896177 & 25.25 & 19655283 & 23.54 & 7054485 & 22.41 \\
\hline & & 0.8 & 20650575 & 33.20 & 4317269 & 24.21 & 2415883 & 21.04 & 2693626 & 20.63 \\
\hline & & 1.0 & 610871 & 34.08 & 1000351 & 26.66 & 1376567 & 22.48 & 1535043 & 19.82 \\
\hline
\end{tabular}


Table 7. (Continued)

\begin{tabular}{|c|c|c|c|c|c|c|c|c|c|c|}
\hline \multirow[t]{2}{*}{ rng } & \multirow[t]{2}{*}{$n$} & \multirow[t]{2}{*}{$T$} & \multicolumn{2}{|c|}{$R=0.2$} & \multicolumn{2}{|c|}{$R=0.4$} & \multicolumn{2}{|c|}{$R=0.6$} & \multicolumn{2}{|c|}{$R=0.8$} \\
\hline & & & NG & $\% \mathrm{LB}$ & NG & $\%$ LB & NG & $\% \mathrm{LB}$ & NG & $\% \mathrm{LB}$ \\
\hline \multirow[t]{18}{*}{$\mathrm{H}$} & \multirow[t]{6}{*}{10} & 0.0 & 164 & 81.35 & 340 & 74.08 & 446 & 66.35 & 632 & 60.62 \\
\hline & & 0.2 & 1822 & 25.68 & 1398 & 37.18 & 790 & 50.20 & 1079 & 46.81 \\
\hline & & 0.4 & 3163 & 21.12 & 3033 & 24.80 & 2420 & 28.55 & 1839 & 34.62 \\
\hline & & 0.6 & 3163 & 37.22 & 2806 & 34.04 & 2416 & 33.87 & 1456 & 33.71 \\
\hline & & 0.8 & 1424 & 48.41 & 1960 & 37.22 & 1592 & 33.26 & 1441 & 33.82 \\
\hline & & 1.0 & 652 & 55.12 & 940 & 47.24 & 1217 & 43.53 & 1092 & 42.30 \\
\hline & \multirow[t]{6}{*}{15} & 0.0 & 2229 & 78.74 & 2236 & 73.81 & 7031 & 63.58 & 9086 & 58.15 \\
\hline & & 0.2 & 128102 & 8.94 & 67324 & 25.97 & 28272 & 34.16 & 37252 & 38.71 \\
\hline & & 0.4 & 231050 & 19.04 & 245601 & 23.92 & 213025 & 23.60 & 109675 & 29.95 \\
\hline & & 0.6 & 336058 & 34.21 & 339098 & 28.52 & 218147 & 23.63 & 87756 & 20.93 \\
\hline & & 0.8 & 167745 & 35.67 & 84906 & 28.25 & 78040 & 26.37 & 68863 & 23.18 \\
\hline & & 1.0 & 17755 & 43.53 & 37175 & 31.47 & 36066 & 28.50 & 34288 & 29.33 \\
\hline & \multirow[t]{6}{*}{20} & 0.0 & 10327 & 79.53 & 43650 & 65.55 & 131308 & 56.71 & 185452 & 52.43 \\
\hline & & 0.2 & 8320655 & 5.26 & 3078434 & 22.36 & 658317 & 33.89 & 561120 & 35.87 \\
\hline & & 0.4 & 13618711 & 20.48 & 28010326 & 24.62 & 39339572 & 22.61 & 12955845 & 24.55 \\
\hline & & 0.6 & 47595664 & 33.30 & 44118348 & 27.65 & 25162764 & 21.68 & 7908324 & 19.16 \\
\hline & & 0.8 & 15643648 & 31.26 & 7958865 & 22.42 & 2802132 & 20.04 & 1887911 & 17.22 \\
\hline & & 1.0 & 398030 & 37.00 & 1218970 & 23.40 & 1049279 & 23.28 & 1006924 & 21.30 \\
\hline
\end{tabular}

bound test for INS $=0.50$. The tardiness factor $T$ has a clear and significant effect on the number of nodes generated. In fact, this number is much lower when $T=0.0$ or $T=1.0$, and then increases significantly as $T$ approaches its intermediate values. Once more, this result is in accordance with the runtimes previously given in Table 5 . The lower bound test eliminates a large percentage of nodes for instances with $T=0.0$. This is to be expected since the lower bound performs particularly well when most jobs are early, as mentioned before. For the remaining parameter values, however, the insertion-based test usually fathoms a larger proportion of the nodes.

\section{Conclusion}

In this paper, we considered the single machine scheduling problem with linear earliness and quadratic tardiness costs, and no machine idle time. We developed a lower bound based on the relaxation of the jobs' completion times. An optimal branch-and-bound algorithm was then presented. This algorithm incorporates the proposed lower bound, as well as an insertion-based fathoming test.

The branch-and-bound procedures were tested on a wide set of randomly generated problems. These algorithms were capable of solving, within reasonable computation times, instances with up to 20 jobs. The best results were obtained by setting the INS parameter required by the insertion dominance procedure to 0.50 or 0.75 . In fact, these values not only provided the lowest average computation times, but were also more consistent. Also, as the instance difficulty, and correspondingly the runtime, increased, the branch-and-bound procedures with INS equal to 0.50 or 
0.75 became increasingly more efficient, since the increase in the runtime was much slower for these procedures.

\section{Acknowledgment}

The author would like to thank the anonymous referees and an associate editor for several, and most useful, comments and suggestions that were used to improve this paper.

\section{References}

Azizoglu, M, S Kondakci and O Kirca (1991). Bicriteria scheduling problem involving total tardiness and total earliness penalties. International Journal of Production Economics, 23, 17-24.

Baker, KR and GD Scudder (1990). Sequencing with earliness and tardiness penalties: A review. Operations Research, 38, 22-36.

Garey, MR, RE Tarjan and GT Wilfong (1988). One-processor scheduling with symmetric earliness and tardiness penalties. Mathematics of Operations Research, 13, 330-348.

Gupta, SK and T Sen (1983). Minimizing a quadratic function of job lateness on a single machine. Engineering Costs and Production Economics, 7, 187-194.

Kanet, JJ and V Sridharan (2000). Scheduling with inserted idle time: Problem taxonomy and literature review. Operations Research, 48, 99-110.

Kim, YD and CA Yano (1994). Minimizing mean tardiness and earliness in single-machine scheduling problems with unequal due dates. Naval Research Logistics, 41, 913-933.

Korman, K (1994). A pressing matter. Video, 46-50.

Landis, K (1993). Group technology and cellular manufacturing in the Westvaco Los Angeles VH department. Project report in IOM 581, School of Business, University of Southern California.

Schaller, J (2002). Minimizing the sum of squares lateness on a single machine. European Journal of Operational Research, 143, 64-79.

Schaller, J (2004). Single machine scheduling with early and quadratic tardy penalties. Computers \&s Industrial Engineering, 46, 511-532.

Sen, T, P Dileepan and MR Lind (1995). Minimizing a weighted quadratic function of job lateness in the single machine system. International Journal of Production Economics, 42, 237-243.

$\mathrm{Su}, \mathrm{L}-\mathrm{H}$ and P-C Chang (1998). A heuristic to minimize a quadratic function of job lateness on a single machine. International Journal of Production Economics, 55, 169-175.

Sun, X, JS Noble and CM Klein (1999). Single-machine scheduling with sequence dependent setup to minimize total weighted squared tardiness. IIE Transactions, 31, $113-124$

Taguchi, G (1986). Introduction to Quality Engineering. Asian Productivity Organization, Tokyo, Japan.

Valente, JMS (2006). Heuristics for the single machine scheduling problem with early and quadratic tardy penalties. European Journal of Industrial Engineering, 1, 431-448.

Valente, JMS (2007). An exact approach for single machine scheduling with quadratic earliness and tardiness penalties. Working Paper 238, Faculdade de Economia, Universidade do Porto, Portugal.

Wagner, BJ, DJ Davis and H Kher (2002). The production of several items in a single facility with linearly changing demand rates. Decision Sciences, 33, 317-346. 
Jorge M. S. Valente is Assistant Professor in the Management Department of the Faculty of Economics, University of Porto (Portugal). He holds a Ph.D. degree in Management Science from the University of Porto. He has published in Asia-Pacific Journal of Operational Research, Computers \& Industrial Engineering, Computers \& Operations Research, International Journal of Production Economics, Journal of Manufacturing Systems and in the Journal of the Operational Research Society, among others. His current research interests include production scheduling, combinatorial optimization, heuristic techniques and agent-based computational economics. 